\title{
Development of a constitutive model of soft tissues for FE analyses using a bottom-up approach
}

\author{
J. Vychytil ${ }^{a, *}$, M. Holeček ${ }^{a}$

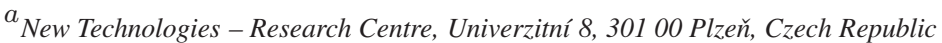

Received 10 November 2016; accepted 21 August 2018

\begin{abstract}
This paper presents the development of a two-scale anisotropic hyperelastic material model whose microstructure is motivated by the arrangement of soft tissues. In a bottom-up approach, we start at the microscale, identifying the components that are relevant for our model. These components are represented by simplified mechanical elements, such as linear springs and incompressible volumes. The next step is to use the concept of the representative volume element connecting the micro- and macroscales. Introducing principal material directions, the notion of invariants and pseudo-invariants is employed to derive a formula for the strain energy function. In fact, two hyperelastic models are proposed. In the simplified one, the microstructure is formed of a network of linear springs. In the second one, an incompressible volume is added to the representation of the microstructure. This results in the model's having a nonlinear response, with the strain energy function arising as a solution to a minimization problem. The properties of the strain energy function and the influence of anisotropy are demonstrated on a simple tension test and a simple shear test. Applications of the proposed model to the description of prestressed materials, non-affine deformations, and real tissue modelling are presented.
\end{abstract}

(c) 2018 University of West Bohemia. All rights reserved.

Keywords: constitutive model, soft tissues, RVE, nonlinear response

\section{Introduction}

A very important task of recent continuum thermomechanics is the formation of continuum models of highly complex materials with a strong dependence on their microscopic arrangement. A typical example is living tissue. The crucial step in forming continuum models is the way in which the microscopic structure of the material is averaged. This means in fact that a huge number of microscopic degrees of freedom are 'neglected', so that only a few relevant 'modes' survive in the intended macroscopic continuum model. The important task of continuum thermomechanics is therefore not only the formation of sophisticated continuum models, but also searching for the relevant features of the microscopic structures that are responsible for various modes of the macroscopic behaviour of the particular material. Concerning biological tissues, a noticeable feature of their microscopic arrangement is their having various conglomerates of fibres. This causes an inevitable anisotropy in the whole-scale hierarchy.

Continuum models of anisotropic materials have been addressed for a couple of decades. In continuum biomechanics, for example, improved material models based on polynomial [31] and logarithmic strain energy functions [28] have been proposed to describe the deformations of an arterial wall. The anisotropy is here taken into account via a dependency on the components of the Green strain tensor. In a similar way, a two-dimensional model based on an exponential

\footnotetext{
*Corresponding author. Tel.: +420 377634 838, e-mail: jvychyti@ntc.zcu.cz. https://doi.org/10.24132/acm.2018.330
} 
form of strain energy function is proposed by [8]. It is generalized for a three-dimensional case [4] and shear deformations [6]. All these models are phenomenological, which means they are determined by empirical formulae of strain energy functions proposed to fit the experimental data. They are suitable for the description of an overall macroscopic mechanical response, taking anisotropy into account. However, they lack information regarding the origin, in the microscale, of the macroscopic anisotropy. Hence, the identification of the material constants appearing in phenomenological models is not an easy task.

New approaches have been developed in order to formulate more general, improved, continuum models. For instance, the arrangement of the microstructure in terms of preferred fibre directions is coupled with the overall mechanical response in what are known as structural models [14]. Here, the notion of invariants and pseudo-invariants is used [17,26]. This idea is based on the fact that the mechanical response of isotropic materials can be expressed using the invariants of the right Cauchy-Green strain tensor, see, e.g. [2]. In the case of anisotropic materials, such as in fibre-reinforced solids, additional information is needed. Therefore, additional invariants (sometimes called pseudo-invariants) are introduced using structural tensors. The physical interpretation of some of the pseudo-invariants is straightforward, such as with the stretches along the preferred directions.

Models of fibre-reinforced materials are often used to capture the anisotropy of biological tissues, as well as engineering materials such as composites. In this case, the strain energy is a function of both invariants and pseudo-invariants relating the overall mechanical response to the arrangement of microstructure. [14], for instance, proposes a hyperelastic model suitable for the description of an arterial wall. In this case, the angle between the preferred fibre directions appears as a structural parameter. The description of an arterial wall is also the object of [15], where the Gent model is generalized to anisotropic materials. In [23], homogenization techniques are used to propose a multiscale model for the mechanical response of vein walls. In rubberlike materials, polymeric fibres are governing the mechanical response. In [36], single fibre is represented with a wormlike chain model. Mechanical response of an isotropic network is then derived as well as its simplification forming three-chain and eight-chain model. Its generalization for transversely isotropic materials is presented in [20]. Here, the idea of pseudo-invariants is employed to propose a hyperelastic model of soft tissue based on a wormlike chain model of a single collagen fibril. The resulting model is successfully applied to the description of deformations of rabbit skin and the effect of remodeling. The wormlike chain model is also employed in [21] to propose a strain energy function of soft tissue in a decoupled form. The development of a structural model of soft tissue taking into account plastic deformations is described in [29]. In the field of viscoelasticity, a transversely isotropic model is proposed in [22] to describe soft connective tissue. An example of a bottom-up approach in a model is proposed in [24]. Starting from the arrangement of fibrils within fascicles, a constitutive model of ligaments and tendons is derived. A coupling of the atomistic approach and the continuum approach is provided in [3]. There, a continuum model of collagen is based on an atomistic approach with the help of molecular dynamics. An application of structural models to the description of skeletal muscles is given in, for instance, $[1,16]$. Engineering materials are not to be forgotten: for the application of the theory to the description of composites see, e.g. [13].

The aim of the present paper is to propose a two-scale hyperelastic anisotropic model suitable for finite element (FE) analysis. Using a generalized continuum approach, the model captures some aspects of soft tissues. It allows including possible non-affine behaviour at the microscale. That is, the arrangement of the microstructure is driven by a local energy minimizing principle and is not governed directly by the macroscopic deformation gradient. Also, prestress can be 
included in the reference configuration. In fact, the new model is based on a model previously presented in $[11,34]$, which was proposed to describe certain deformation states, such as the states of an arterial segment undergoing an inflation-deflation test [35]. Here, we propose a generalization, presenting a material model which is suitable for FE analysis of an arbitrary deformation state. This is done simply by adding diagonal elastic elements within the RVE of the previous model. Adopting the notion of pseudo-invariants, the strain energy function of the macroscopic body is obtained.

This paper is organized as follows. In Section 2, the concept of structural tensors is explained according to [17]. Considering an orthotropic material with three principal material directions, three structural tensors as well as pseudo-invariants $I_{41}, \ldots, I_{x 23}$ are introduced. The notion of the representative volume element is explained. These ideas are employed in Section 3 to propose a simple anisotropic model with an RVE formed of a network of linear springs. The effect of anisotropy is shown using a simple tension test. The final material model is proposed in Section 4. It can be understood as both an improvement of the simple model as well as a generalization of the model proposed in [11]. It is a two-scale hyperelastic anisotropic model which allows including a possible non-affine behaviour of the microstructure. Characterized by the strain energy as a function of the pseudo-invariants, the model is subjected to simple tension and simple shear tests. However, it is suitable for an FE study of an arbitrary deformation state. Section 5 focuses on the abilities of the proposed model regarding non-affine behaviour at the microscale, prestress, and its application to real-tissue modelling.

\section{Theoretical background}

\subsection{Concept of pseudo-invariants}

The mechanical response of hyperelastic materials is often characterized via the strain energy function,

$$
W=W(\mathbf{C}),
$$

where $\mathbf{C}$ is the right Cauchy-Green strain tensor. In the case of isotropic materials, $W$ must be a function only of the invariants of $\mathbf{C}$ such as

$$
W(\mathbf{C})=W\left(I_{1}, I_{2}, I_{3}\right),
$$

see, e.g. [2] for details.

In anisotropic materials, the notion of pseudo-invariants is often used. Some details can be found, for instance, in $[14,19,22,25]$. In the present paper we focus on orthotropic materials, i.e. materials in which the mechanical properties are governed by three mutually perpendicular directions. We follow the approach described in [17]. As a representation of principal material directions, let us introduce three unit vectors, $\boldsymbol{n}_{\mathbf{1}}, \boldsymbol{n}_{\mathbf{2}}$ and $\boldsymbol{n}_{\mathbf{3}}$, which form an orthonormal basis. We define three structural tensors:

$$
\mathrm{N}_{1}=n_{1} \otimes n_{1}, \quad \mathrm{~N}_{2}=n_{2} \otimes n_{2}, \quad \mathrm{~N}_{3}=n_{3} \otimes n_{3} .
$$

The following pseudo-invariants are then introduced:

$$
\begin{aligned}
I_{41} & =\mathbf{C}: \mathbf{N}_{\mathbf{1}}, & I_{42} & =\mathbf{C}: \mathbf{N}_{\mathbf{2}}, & I_{43} & =\mathbf{C}: \mathbf{N}_{\mathbf{3}}, \\
I_{x 12} & =\boldsymbol{n}_{\mathbf{1}} \cdot \mathbf{C} \boldsymbol{n}_{\mathbf{2}}, & I_{x 13} & =\boldsymbol{n}_{\mathbf{1}} \cdot \mathbf{C} \boldsymbol{n}_{\mathbf{3}}, & I_{x 23} & =\boldsymbol{n}_{\mathbf{2}} \cdot \mathbf{C} \boldsymbol{n}_{\mathbf{3}} .
\end{aligned}
$$

The mechanical response of an orthotropic material is dependent on the principal material directions. In analogy to isotropic materials, this can be expressed in terms of both the invariants 
and the pseudo-invariants. Since $I_{43}$ and $I_{x 23}$ can be omitted [12], the strain energy function is expressed as

$$
W\left(\mathbf{C}, \boldsymbol{n}_{\mathbf{1}}, \boldsymbol{n}_{\mathbf{2}}, \boldsymbol{n}_{\mathbf{3}}\right)=W\left(I_{1}, I_{2}, I_{3}, I_{41}, I_{42}, I_{x 12}, I_{x 13}\right)
$$

\subsection{Representative volume element}

The idea of a representative volume element (RVE) consists in associating a continuum point $\boldsymbol{x}$ with a certain volume element [7,18], as is depicted in Fig. 1. The volume element contains components that represent the microstructure of the material under consideration, such as fibres, inclusions, particles, etc. If affine deformations are considered, the configuration of the microstructure is given directly by the macroscopic deformation gradient, as described, e.g. in $[9,10]$. Considering non-affine deformations, the linkage between the configuration of the microstructure and the macroscopic deformation gradient is not straightforward, see, e.g. [11]. However, the notion of the RVE links the deformation at both the macro- and microscale in both cases. The strain energy function of the macroscopic body $W(\mathbf{C}(\boldsymbol{x}))$ can thus be derived based on the knowledge of the mechanical response of the individual constituents embedded within the RVE.

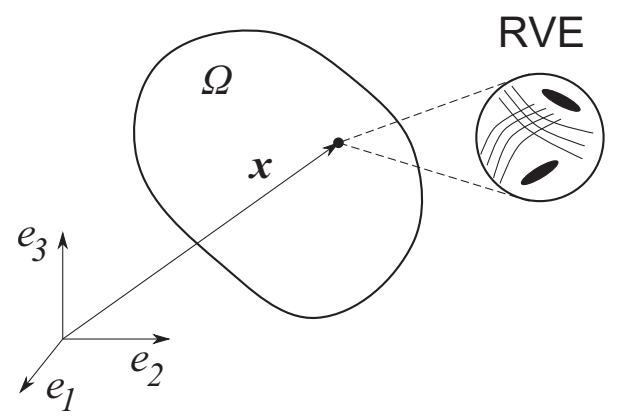

Fig. 1. Representative volume element associated with a continuum point $\boldsymbol{x}$

\section{Pseudo-invariants in an anisotropic model}

\subsection{Model proposal}

In this example, the notion of RVE and pseudo-invariants is adopted to propose a hyperelastic anisotropic model. Its microstructure is formed of springs which represent fibres aligned with preferred directions, see Fig. 2. The RVE has the shape of a rectangular body defined by the vectors $c_{i}$ at the reference state,

$$
\boldsymbol{c}_{\mathbf{1}}=c_{1} \boldsymbol{n}_{\mathbf{1}}, \quad \boldsymbol{c}_{\mathbf{2}}=c_{2} \boldsymbol{n}_{\mathbf{2}}, \quad \boldsymbol{c}_{\mathbf{3}}=c_{3} \boldsymbol{n}_{\mathbf{3}}
$$

The microstructure is formed of 7 linear springs. Three orthogonal springs have the rigidities of $K_{i}^{c}$ and the rest lengths of $c_{i}^{r e s t}, i=1,2,3$. The diagonal springs have the rigidities of $K_{j}^{d}=K^{d}$ and the rest lengths of $d_{j}^{r e s t}, j=1, \ldots, 4$. To ensure a stress-free structure in the reference state, let us choose

$$
c_{i}^{r e s t}=c_{i}, \quad d_{j}^{r e s t}=d=\sqrt{c_{1}^{2}+c_{2}^{2}+c_{3}^{2}} .
$$

In the current state, the RVE takes the shape of a rhomboidal-rectangular body. Considering affine deformations of the microstructure, the RVE is described as

$$
c_{1}^{\prime}=\mathbf{F} c_{1}, \quad c_{2}^{\prime}=\mathbf{F} c_{2}, \quad c_{3}^{\prime}=\mathbf{F} c_{3}
$$



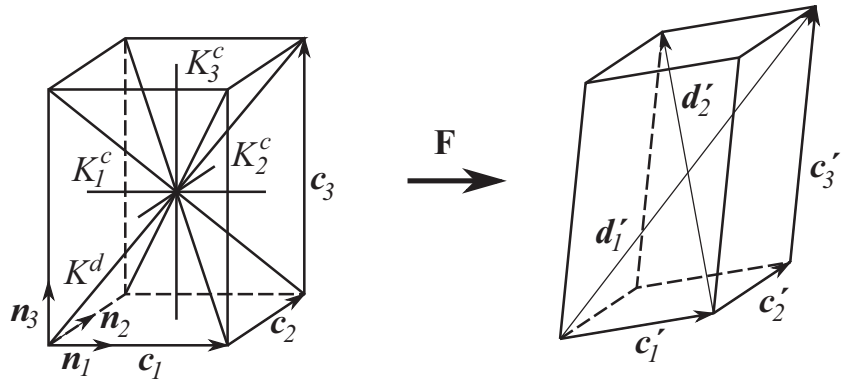

Fig. 2. Representative volume element of the model in both the reference configuration (left) and the current configuration (right)

see Fig. 2. Let us denote with $\boldsymbol{d}_{j}^{\prime}$ vectors describing deformed diagonal springs such as

$$
d_{1}^{\prime}=c_{1}^{\prime}+c_{2}^{\prime}+c_{3}^{\prime}, \quad d_{2}^{\prime}=-c_{1}^{\prime}+c_{2}^{\prime}+c_{3}^{\prime}, \quad \text { etc. }
$$

The energy of the RVE in the current configuration is then given by a sum of quadratic contributions,

$$
E_{R V E}=\sum_{i} \frac{K_{i}^{c}}{2}\left(\left\|\boldsymbol{c}_{\boldsymbol{i}}^{\prime}\right\|-c_{i}\right)^{2}+\frac{K^{d}}{2} \sum_{j}\left(\left\|\boldsymbol{d}_{\boldsymbol{j}}^{\prime}\right\|-d\right)^{2},
$$

where $\left\|\boldsymbol{c}_{\boldsymbol{i}}^{\prime}\right\|$ are the current lengths of the orthogonal springs and $\left\|\boldsymbol{d}_{\boldsymbol{j}}^{\prime}\right\|$ are the current lengths of the diagonal springs.

The strain energy function of the macroscopic body is given only by the contribution of extended/compressed springs at the microscale. That is,

$$
W=\frac{E_{R V E}}{V_{R V E}}, \quad V_{R V E}=c_{1} c_{2} c_{3},
$$

where $V_{R V E}$ is the volume of the RVE. In such a definition, $W$ is the Helmholtz free energy function per unit reference volume, see, e.g. [12]. Following the approach described in Section 2.1, the current lengths of the springs can be expressed as functions of the pseudo-invariants. Hence, the strain energy function can be expressed as

$$
\begin{aligned}
W\left(I_{1}, \ldots, I_{x 23}\right) & =W_{\text {ort }}\left(I_{41}, I_{42}, I_{43}\right)+W_{\text {diag }}\left(I_{41}, \ldots, I_{x 23}\right), \\
W_{\text {ort }} & =\frac{1}{2 V_{R V E}} \sum_{i=1}^{3} K_{i}^{c} c_{i}^{2}\left(\sqrt{I_{4 i}}-1\right)^{2}, \\
W_{\text {diag }} & =\frac{K^{d}}{2 V_{R V E}} \sum_{j=1}^{4}\left(\sqrt{\sum_{i=1}^{3} I_{4 i} c_{i}^{2}+p_{j} 2 c_{1} c_{2} I_{x 12}+q_{j} 2 c_{1} c_{3} I_{x 13}+r_{j} 2 c_{2} c_{3} I_{x 23}-d}\right)^{2} .
\end{aligned}
$$

Here, $W_{\text {ort }}$ represents the contribution of the orthogonal springs and $W_{\text {diag }}$ the contribution of the diagonal springs. The coefficients are

$$
\begin{aligned}
& p_{1}=1, \quad p_{2}=-1, \quad p_{3}=1, \quad p_{4}=-1, \\
& q_{1}=1, \quad q_{2}=-1, \quad q_{3}=-1, \quad q_{4}=1, \\
& r_{1}=1, \quad r_{2}=1, \quad r_{3}=-1, \quad r_{4}=-1 .
\end{aligned}
$$

If we assume the representative volume element to be filled with an isotropic matrix, the corresponding contribution appears in (11). For instance, consider the matrix as a compressible neo-Hookean material defined by the strain energy function

$$
W_{i s o}\left(I_{1}, I_{3}\right)=\frac{\mu}{2}\left(I_{1}-3\right)+\frac{\mu}{2 \beta}\left(I_{3}^{-\beta}-1\right),
$$


see, e.g. [20] for details. The expression (11) is then modified as

$$
W\left(I_{1}, \ldots, I_{x 23}\right)=W_{\text {iso }}\left(I_{1}, I_{3}\right)+W_{\text {ort }}\left(I_{41}, I_{42}, I_{43}\right)+W_{\text {diag }}\left(I_{41}, \ldots, I_{x 23}\right) .
$$

The proposed model has in fact a similar structure to the network model presented in [36]. In our model, the elasticity is represented with seven linear springs while the wormlike chain model is used in [36] to capture the non-linear mechanical response typical for rubber-like materials. In both approaches, affine deformation is considered, that is, distorsion and rotation of individual fibres is governed by macroscopic deformation gradient. However, we employ springs of various parameters resulting in an anisotropic model. In [36], on the other hand, combination of the three-chain and eight-chain model represents a simplification of the isotropic network model.

\subsection{Simple tension test}

To illustrate the effect of anisotropy, a simple tension test is analyzed. We assume incompressibility and transverse isotropy, i.e. the material properties are identical for the first and second principal directions. Hence, the deformation gradient and the first Piola-Kirchhoff stress tensor take the form

$$
\mathbf{F}=\left(\begin{array}{ccc}
\frac{1}{\sqrt{\lambda}} & 0 & 0 \\
0 & \frac{1}{\sqrt{\lambda}} & 0 \\
0 & 0 & \lambda
\end{array}\right), \quad \boldsymbol{\Pi}=\left(\begin{array}{ccc}
0 & 0 & 0 \\
0 & 0 & 0 \\
0 & 0 & \Pi_{33}
\end{array}\right)
$$

The constitutive equations of an incompressible hyperelastic material are

$$
\boldsymbol{\Pi}=\frac{\partial W}{\partial \mathbf{F}}-p \mathbf{C o f} \mathbf{F}
$$

where $p$ is the hydrostatic pressure and Cof $\mathbf{F}$ is the cofactor matrix, see, e.g. [12]. For each component of the stress tensor, we obtain

$$
0=\frac{\partial W}{\partial F_{11}}-p \frac{1}{F_{11}}, \quad 0=\frac{\partial W}{\partial F_{22}}-p \frac{1}{F_{22}}, \quad \Pi_{33}=\frac{\partial W}{\partial F_{33}}-p \frac{1}{F_{33}}
$$

We use

$$
\boldsymbol{n}_{\mathbf{1}}=(1,0,0), \quad \boldsymbol{n}_{\mathbf{2}}=(0,1,0), \quad \boldsymbol{n}_{\mathbf{3}}=(0,0,1)
$$

to specify the invariants $I_{41}, \ldots, I_{x 23}$ according to (2) and (3). Hence, the strain energy (11) can be understood as a function

$$
W\left(I_{41}, \ldots, I_{x 23}\right)=f(\lambda)
$$

Using (17), the component of the first Piola-Kirchhoff stress tensor can be expressed as

$$
\Pi_{33}=\frac{\partial W}{\partial F_{33}}+\frac{\mathrm{d} F_{11}}{\mathrm{~d} F_{33}} \frac{\partial W}{\partial F_{11}}+\frac{\mathrm{d} F_{22}}{\mathrm{~d} F_{33}} \frac{\partial W}{\partial F_{22}}=\frac{\mathrm{d} W}{\mathrm{~d} F_{33}}=\frac{\mathrm{d} W}{\mathrm{~d} \lambda} .
$$

Finally, we use the relation between the first Piola-Kirchhoff stress tensor $\Pi$ and the Cauchy stress tensor $\sigma$,

$$
\mathbf{\Pi}=\operatorname{det} \mathbf{F} \boldsymbol{\sigma} \mathbf{F}^{-T},
$$

see, e.g. [2]. The component of the Cauchy stress tensor (normal stress) is then determined by

$$
\sigma=\lambda \frac{\mathrm{d} f(\lambda)}{\mathrm{d} \lambda}
$$


Choose the parameters

$$
K=\frac{K_{1}^{c}}{c_{1}}=1 \mathrm{MPa}, \quad k_{13}^{*}=\frac{K_{1}^{c}}{K_{3}^{c}}=1, \quad k_{1 d}^{*}=\frac{K_{1}^{c}}{K^{d}}=1 .
$$

The direction of tension is aligned with the third principal direction of material axes, i.e. the preferred orientation of the transversely isotropic model, see Fig. 3. As a measure of the anisotropy, we choose the parameter

$$
c_{13}^{*}=\frac{c_{1}}{c_{3}} .
$$

The dependency (22), i.e. the stress-stretch characteristics for varying parameter $c_{13}^{*}$, is depicted in Fig. 3. The result suggest that the value of the parameter $c_{13}^{*}$ has a significant effect on the overall mechanical response.

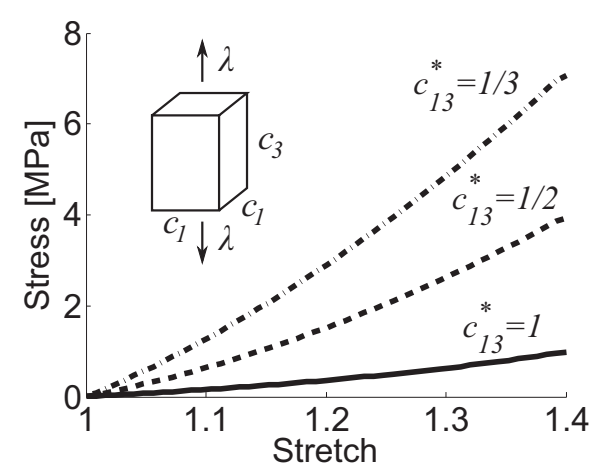

Fig. 3. Stress-stretch curves for various values of the ratio $c_{13}^{*}$. The direction of tension is indicated

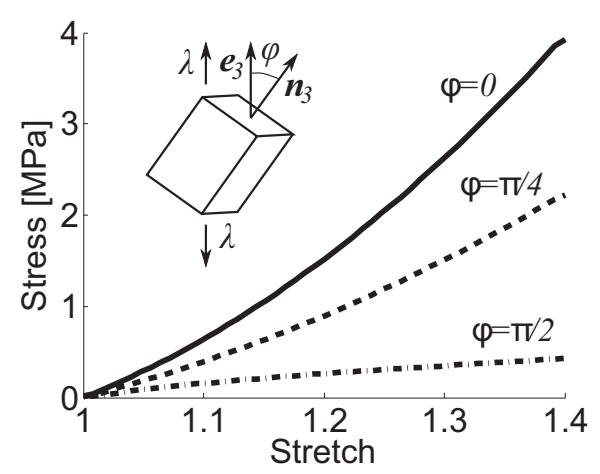

Fig. 4. Stress-stretch curves for varying rotation of the RVE within material. Direction of tension is indicated

Second, the influence of the angle $\varphi$ between the principal material direction and the direction of tension will be studied, see Fig. 4. This is the case when the RVE is not aligned with the direction of tension while the ratio $c_{13}^{*}$ is fixed, $c_{13}^{*}=1 / 2$. The deformation gardient is constrained to be of the diagonal form given in (15). That is, we constrain the same deformation of the body for any rotation of principal material direction. Such deformation of anisotropic material with general angle $\varphi$ is no longer connected with an uniaxial tension. In fact, shear stress is induced. In Fig. 4, however, the component of the Cauchy stress tensor corresponding to the normal stress is plotted. Again, a significant effect of the angle on the mechanical response is illustrated.

Lastly, the ability of the model to include prestress, i.e. the pre-existing tension within springs in the reference configuration, is demonstrated. Assume that the rest lengths of the three orthogonal springs satisfy $c_{i}^{\text {rest }} \neq c_{i}$. In other words, these springs are extended at the reference state and carry a pre-existing tension. Therefore, the term $c_{i}$ must be replaced with $c_{i}^{\text {rest }}$ in (9). Introducing the prestress parameters

$$
P_{i}=1-\frac{c_{i}^{r e s t}}{c_{i}}
$$

the resulting contribution $W_{\text {ort }}$ in (11) then becomes

$$
W_{\text {ort }}=\frac{1}{2 V_{R V E}} \sum_{i=1}^{3} K_{i}^{c} c_{i}^{2}\left(\sqrt{I_{4 i}}-1+P_{i}\right)^{2} .
$$




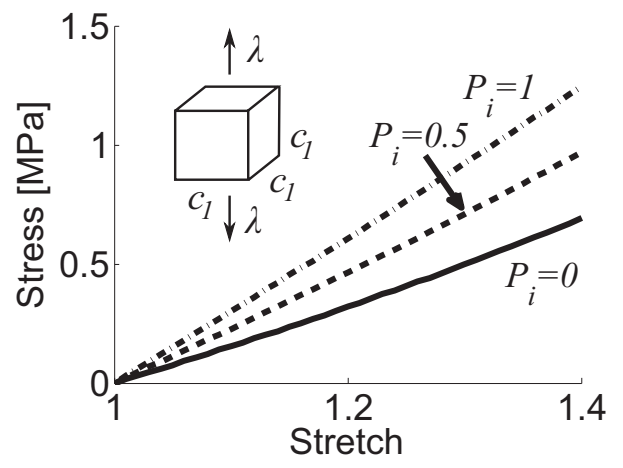

Fig. 5. Stress-stretch curves for varying level of prestress. Direction of tension is indicated

The influence of the prestress on the overall mechanical response is illustrated in Fig. 5. In this case, the material parameters are chosen according to (23). However, we assume $K^{d}=0 \mathrm{MPa}$, $c_{13}^{*}=1$ and $k_{1 d}^{*}$ is not defined. With increasing prestress, the stiffness of the model increases. This effect, known as prestress-induced stiffening, is in agreement with experimental observations for living cells, see, e.g. [27].

\section{Anisotropic material model for FE analyses}

\subsection{Microstructure}

The proposed model is based on an arrangement of soft tissue, specifically, smooth muscle tissue. The aim is to focus on certain features of the mechanical response rather than to propose a realistic model of smooth muscle tissue. Restricting considerations to the elasticity only, the model has a cellular structure reinforced with cytoskeleton. Consider the smooth muscle tissue as a regular composition of living cells, as depicted in Fig. 6. The cells are closely attached to each other via an extracellular matrix. Each cell is reinforced with a network of polymer fibres, known as a cytoskeleton, which is responsible for the mechanical response of the cell. In the model, each cell is formed of an incompressible volume in the shape of a rectangular body, see Fig. 6. The cytoskeleton is represented by a network of linear springs. The elasticity of the extracellular matrix is also represented by linear springs that interconnect mutually neighbouring cells.

An RVE as depicted in Fig. 7 was chosen. It has the shape of a rectangular body with sizes $l_{1}$, $l_{2}$ and $l_{3}$ and it contains one incompressible volume and interconnecting springs. In itself, each incompressible volume has a structure corresponding to the example presented in Section 3.
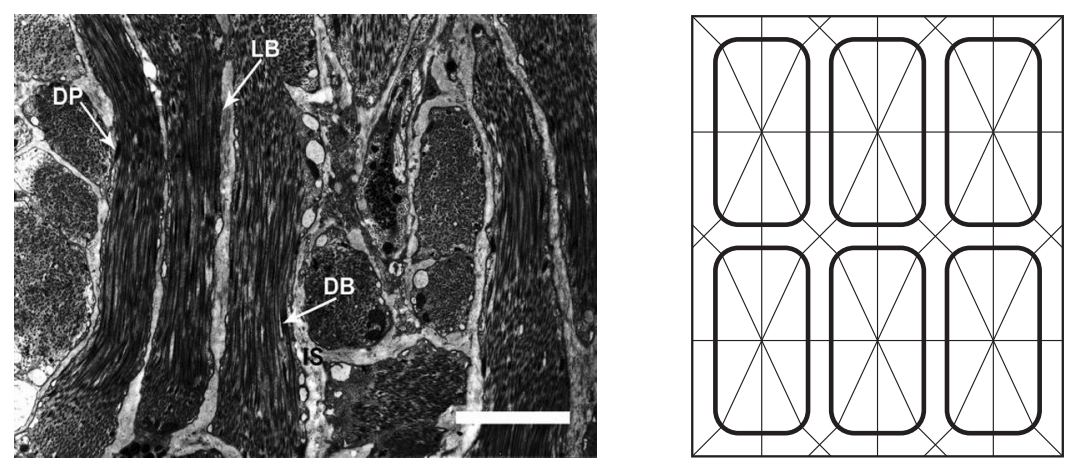

Fig. 6. Cellular structure of real tissue (left [30]) and its model representation (right) in a two-dimensional projection 


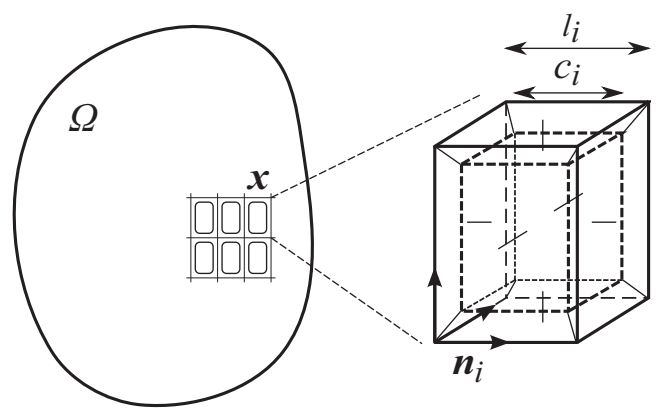

Fig. 7. Each RVE contains one incompressible volume as well as interconnecting springs

The same notation, $K_{j}^{d}, j=1, \ldots, 4$, is used for taking into consideration various rigidities of the diagonal springs. Incompressibility can be expressed using the condition

$$
c_{1} c_{2} c_{3}=V_{c}=\text { const. }
$$

Assume first that there is no prestress within the springs reinforcing the volume at the reference state, that is, dimensions and rest lengths are related according to (6). Concerning the extracellular matrix, there are two extra types of springs. The orthogonal ones have the rigidities $K_{i}^{\Delta}$ and rest lengths $\Delta_{i}^{\text {rest }}=\Delta_{i}, i=1,2,3$, diagonal ones have the rigidities $K_{j}^{\beta}$ and the rest lengths of $\beta_{j}^{r e s t}, i=j, \ldots, 4$. To ensure that the whole structure is stress-free at the reference state, we assume

$$
l_{i}=c_{i}+\Delta_{i}, \quad \beta_{j}^{r e s t}=\beta=\sqrt{\Delta_{1}^{2}+\Delta_{2}^{2}+\Delta_{3}^{2}} .
$$

\subsection{Strain energy function of the macroscopic body}

Introduce three orthonormal vectors $n_{1}, n_{2}, n_{3}$ that represent the principal material directions. Describing the reference state of the model's microstructure with vectors $\boldsymbol{v}_{\boldsymbol{i}}, \boldsymbol{c}_{\boldsymbol{i}}, \boldsymbol{\Delta}_{\boldsymbol{i}}, \boldsymbol{d}_{\boldsymbol{j}}, \boldsymbol{\beta}_{\boldsymbol{j}}$, $i=1,2,3, j=1, \ldots, 4$ (see Fig. 8 ), it is

$$
\boldsymbol{v}_{\boldsymbol{i}}=l_{i} \boldsymbol{n}_{\boldsymbol{i}}, \quad \boldsymbol{c}_{\boldsymbol{i}}=c_{i} \boldsymbol{n}_{\boldsymbol{i}}, \quad \boldsymbol{\Delta}_{\boldsymbol{i}}=\boldsymbol{v}_{\boldsymbol{i}}-\boldsymbol{c}_{\boldsymbol{i}} .
$$

In the current state, the the springs are extended or compressed and the vectors take the form $\boldsymbol{v}_{\boldsymbol{i}}^{\prime}$, $\boldsymbol{c}_{\boldsymbol{i}}^{\prime}, \boldsymbol{\Delta}_{\boldsymbol{i}}^{\prime}, \boldsymbol{d}_{j}^{\prime}$ and $\boldsymbol{\beta}_{j}^{\prime}$, see Fig. 8. With an unknown configuration of the microstructure (position and shape of the inner volume), these vectors are arbitrary in general. Therefore, we make the following assumption to simplify the model. We assume $\boldsymbol{v}_{\boldsymbol{i}}^{\prime}, \boldsymbol{c}_{\boldsymbol{i}}^{\prime}$ and $\boldsymbol{\Delta}_{i}^{\prime}$ are collinear, i.e.

$$
\boldsymbol{v}_{\boldsymbol{i}}^{\prime}=l_{i}^{\prime} \boldsymbol{n}_{\boldsymbol{i}}^{\prime}, \quad \boldsymbol{c}_{\boldsymbol{i}}^{\prime}=c_{i}^{\prime} \boldsymbol{n}_{\boldsymbol{i}}^{\prime}, \quad \Delta_{i}^{\prime}=\Delta_{i}^{\prime} \boldsymbol{n}_{\boldsymbol{i}}^{\prime},
$$
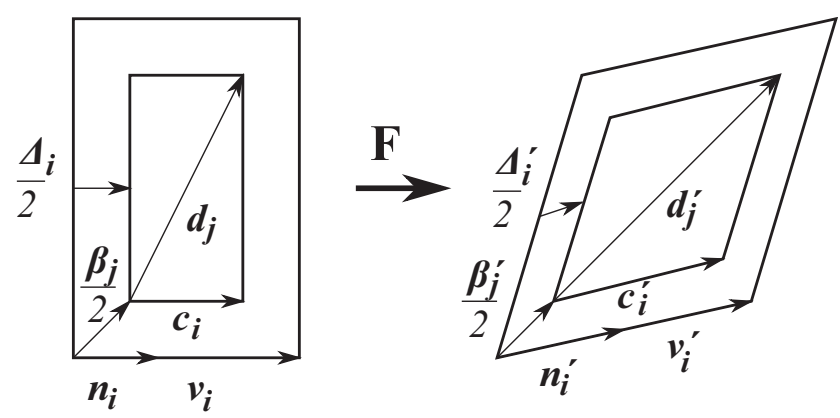

Fig. 8. Reference (left) and a current configuration (right) of the RVE in a 2D projection 
where $\boldsymbol{n}_{\boldsymbol{i}}^{\prime}$ are the vectors $\boldsymbol{n}_{\boldsymbol{i}}$ in the current state and $c_{i}^{\prime}$ are unknown coefficients. Note that

$$
\Delta_{i}^{\prime}=l_{i}^{\prime}-c_{i}^{\prime}
$$

The deformation energy of the whole structure in the current state is given by the quadratic contributions of the individual springs as in (9), i.e

$$
\begin{aligned}
E_{R V E} & =E_{\text {ort }}+E_{\text {diag }}, \\
E_{\text {ort }} & =\sum_{i=1}^{3}\left[\frac{K_{i}^{c}}{2}\left(\left\|\boldsymbol{c}_{\boldsymbol{i}}^{\prime}\right\|-c_{i}\right)^{2}+\frac{K_{i}^{\Delta}}{2}\left(\left\|\boldsymbol{\Delta}_{\boldsymbol{i}}^{\prime}\right\|-\Delta_{i}\right)^{2}\right], \\
E_{\text {diag }} & =\sum_{j=1}^{4}\left[\frac{K_{j}^{d}}{2}\left(\left\|\boldsymbol{d}_{\boldsymbol{j}}^{\prime}\right\|-d\right)^{2}+\frac{K_{j}^{\beta}}{2}\left(\left\|\boldsymbol{\beta}_{\boldsymbol{j}}^{\prime}\right\|-\beta\right)^{2}\right]
\end{aligned}
$$

with $E_{\text {ort }}$ corresponding to the orthogonal springs and $E_{\text {diag }}$ to the diagonal ones. We assume that the shape and dimensions of the RVE are determined directly by the deformation gradient as

$$
\boldsymbol{v}_{i}^{\prime}=\mathbf{F} \boldsymbol{v}_{i}, \quad \boldsymbol{n}_{i}^{\prime}=\mathrm{F} \boldsymbol{n}_{i}
$$

However, there is no such assumption for the inner volume within the RVE. Its shape and dimensions are described by $\boldsymbol{c}_{\boldsymbol{i}}^{\prime}=c_{i}^{\prime} \boldsymbol{n}_{\boldsymbol{i}}^{\prime}$, with $c_{i}^{\prime} \neq c_{i}$ being unknown coefficients. There is no assumption relating vectors $\boldsymbol{c}_{\boldsymbol{i}}^{\prime}$ with the deformation gradient $\mathbf{F}$. In other words, we assume that the macroscopic deformation gradient $\mathbf{F}(\boldsymbol{x})$ determines the overall shape and dimensions of the RVE associated with the point $\boldsymbol{x}$, but there is no such assumption concerning the embedded microstructure. That is, we adopt the idea of non-affine deformations. Initially orthogonal dimensions of the RVE are changing during deformation according to (33). For applications in soft tissues, constant volume of the RVE resulting in incompressible constitutive model is appropriate. In such case, incompressibility has to be enforced with accordance to standard theory, as it is shown in Section 3.2.

We write

$$
\phi=\frac{E_{R V E}}{V_{R V E}}, \quad V_{R V E}=l_{1} l_{2} l_{3}
$$

for the deformation energy of the RVE per unit volume. Using the notion of pseudo-invariants as in Section 3 and introducing the functions

$$
\begin{aligned}
A_{i} & =c_{i}^{\prime} \sqrt{I_{4 i}}-c_{i} \\
B_{i} & =\Delta_{i}^{\prime} \sqrt{I_{4 i}}-\Delta_{i}, \\
C_{j} & =\sqrt{\sum_{i=1}^{3} I_{4 i}\left(c_{i}^{\prime}\right)^{2}+p_{j} 2 c_{1}^{\prime} c_{2}^{\prime} I_{x 12}+q_{j} 2 c_{1}^{\prime} c_{3}^{\prime} I_{x 13}+r_{j} 2 c_{2}^{\prime} c_{3}^{\prime} I_{x 23}-d} \\
D_{j} & =\sqrt{\sum_{i=1}^{3} I_{4 i}\left(\Delta_{i}^{\prime}\right)^{2}+p_{j} 2 \Delta_{1}^{\prime} \Delta_{2}^{\prime} I_{x 12}+q_{j} 2 \Delta_{1}^{\prime} \Delta_{3}^{\prime} I_{x 13}+r_{j} 2 \Delta_{2}^{\prime} \Delta_{3}^{\prime} I_{x 23}-\beta}
\end{aligned}
$$

the deformation energy can be written as

$$
\phi=\frac{1}{2 V_{R V E}}\left[\sum_{i=1}^{3}\left(K_{i}^{c} A_{i}^{2}+K_{i}^{\Delta} B_{i}^{2}\right)+\sum_{j=1}^{4}\left(K_{j}^{d} C_{j}^{2}+K_{j}^{\beta} D_{j}^{2}\right)\right] .
$$


Here, the coefficients $p_{j}, q_{j}$ and $r_{j}$ are defined according to (12). Note that, due to (31) and (35), $\phi$ is a function

$$
\phi=\phi\left(c_{i}^{\prime}, I_{41}, \ldots, I_{x 23}\right),
$$

where $c_{i}^{\prime}$ are the unknown sizes of the volume within the RVE. To determine $c_{i}^{\prime}$, an additional assumption is adopted. In this approach, the idea of energy minimization is employed. As the deformation gradient $\mathbf{F}(\boldsymbol{x})$ determines the shape of the RVE associated with the point $\boldsymbol{x}$, the configuration of the microstructure (the sizes of the embedded volume, $c_{i}^{\prime}$ ) is such that the deformation energy is minimized. Hence, we can finally define the strain energy function as follows:

$$
W\left(I_{41}, \ldots, I_{x 23}\right)=\min _{\substack{c_{i}^{\prime} \\ c_{1}^{\prime} c_{2}^{\prime} c_{3}^{\prime}=V_{c}, c_{i}^{\prime} \leq l_{i}}} \phi\left(c_{i}^{\prime}, I_{41}, \ldots, I_{x 23}\right) .
$$

Although the microstructure is formed of linear springs, the model is nonlinear due to the minimization problem (38). Unfortunately, there is no exact analytical solution and hence the model has to be treated numerically. One option is to find an approximate analytical formula, as in [11] for a simplified model.

\subsection{Simple shear}

As a demonstration of the model, consider the simple shear of a macroscopic body described by a deformation gradient of the form

$$
\mathbf{F}=\left(\begin{array}{lll}
1 & \gamma & 0 \\
0 & 1 & 0 \\
0 & 0 & 1
\end{array}\right)
$$

where $\gamma$ is a constant shear. We use orthonormal basis (18) to specify the invariants $I_{41}, \ldots, I_{x 23}$. The strain energy can be determined using (35), (36) and (38). In fact, it is a function

$$
W\left(I_{41}, \ldots, I_{x 23}\right)=f(\gamma) .
$$

This dependency is plotted in Fig. 9 for a particular choice of the material parameters. Namely,

$$
\begin{aligned}
& \frac{K_{i}^{\Delta}}{l_{i}}=1 e 5 \mathrm{~Pa}, \quad \frac{K_{i}^{c}}{K_{i}^{\Delta}}=0.1, \quad \frac{l_{i}}{l_{j}}=1, \quad \frac{c_{i}}{l_{i}}=1 / 2, \\
& \frac{K_{1}^{\beta}}{l_{1}}=5 e 4 \mathrm{~Pa}, \quad \frac{K_{j}^{\beta}}{K_{l}^{\beta}}=1, \quad \frac{K_{j}^{d}}{K_{j}^{\beta}}=0.1 .
\end{aligned}
$$

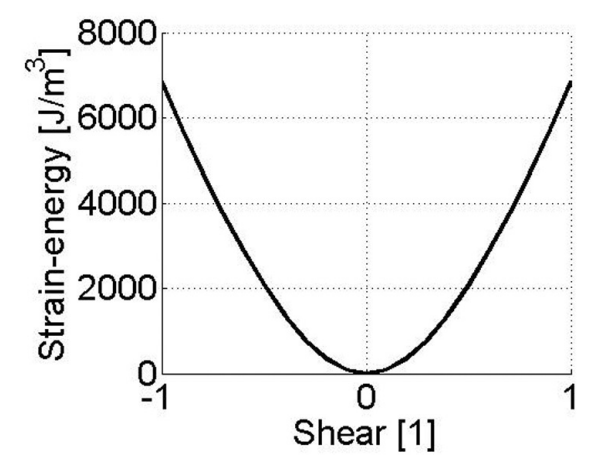

Fig. 9. Dependence of the strain energy on the shear parameter 
According to [12], the strain-energy function should satisfy several conditions. At first, it vanishes in the reference configuration, $W(\mathbf{F}=\mathbf{I})=0$, then it increases with deformation and fulfills $W(\mathbf{F}) \geq 0$. These properties are illustrated in Fig. 9, including the convexity with respect to the shear parameter $\gamma$. However, convexity of the strain energy function with respect to deformation gradient $\mathbf{F}$ is incompatible with the so-called growth condition,

$$
W \rightarrow+\infty \quad \text { if } \quad \operatorname{det} \mathbf{F} \rightarrow+\infty \quad \text { or } \quad \operatorname{det} \mathbf{F} \rightarrow 0
$$

Growth condition means that we would require an infinite amount of strain energy in order to expand a continuum body to the infinite range or to compress it to a point with vanishing volume, see [12]. In our case, the growth condition is fulfilled by the assumption of the incompressible inner volume within the RVE.

\subsection{Simple tension}

Consider a simple tension of a macroscopic body. Assuming incompressible material, the deformation gradient is of the form (15). We use orthonormal basis (18) to specify the invariants $I_{41}, \ldots, I_{x 23}$. The strain energy can be determined using (35), (36) and (38) as a function of stretch parameter $\lambda$ according to relation (19) as it is detailed in section 3.2. The component of the Cauchy stress $\sigma(\lambda)$ is then obtained according to formula (22).

The dependencies of both the strain energy and the component of the Cauchy stress tensor as functions of the stretch $\lambda$ are plotted in Fig. 10 for the material parameters (41). The strain energy fulfills the conditions listed in Section 4.3, including convexity with respect to the parameter $\lambda$. The stress-stretch characteristics reflect the nonlinear response of the material, known as strain-hardening, which is typical for soft tissues, see, e.g. [14].
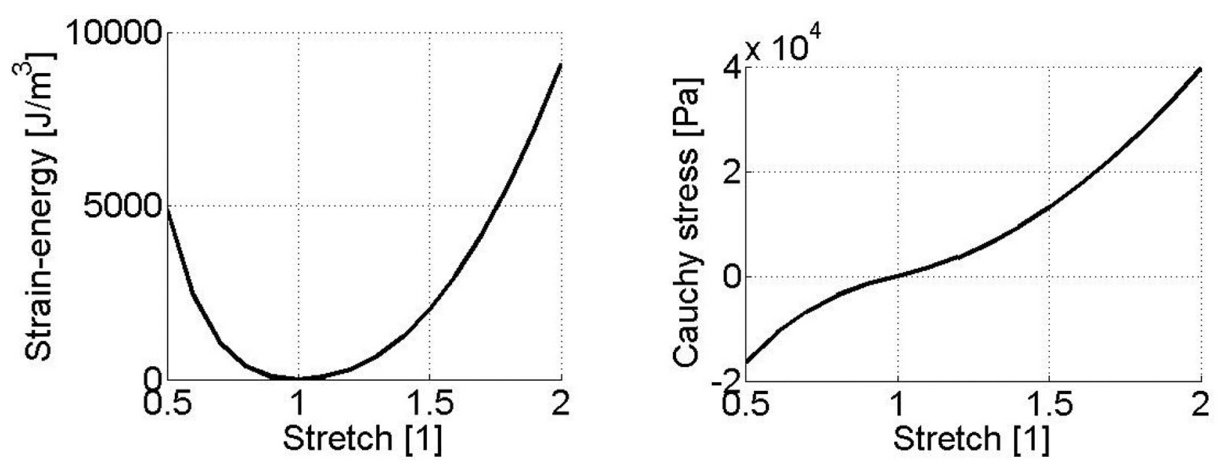

Fig. 10. Dependence of the strain energy (left) and the component of the Cauchy stress (right) on the level of stretch

\section{Abilities of the proposed model}

For the applications presented in this section, consider a restriction of the model. Being reinforced only with the orthogonal springs in the principal directions, it is $K_{j}^{d} \rightarrow 0, K_{j}^{\beta} \rightarrow 0$. Moreover, only special deformation states are studied: those with deformation gradients of the form

$$
\mathbf{F}=\left(\begin{array}{ccc}
\lambda_{1} & 0 & 0 \\
0 & \lambda_{2} & 0 \\
0 & 0 & \lambda_{3}
\end{array}\right)
$$


where the principal stretches are aligned with the principal material directions, i.e.

$$
\mathbf{F} \boldsymbol{n}_{\boldsymbol{i}}=\lambda_{i} \boldsymbol{n}_{\boldsymbol{i}} .
$$

Such a restriction of the model and deformation gradient has already been studied. Each of the mentioned applications has been addressed in individual and detailed papers. In this section, we provide brief descriptions with the corresponding references.

\subsection{Non-affine deformations}

Consider a uniaxial compression of a macroscopic body described by a deformation gradient of the form (15). In fact, this is a reproduction of an example studied in [11], with a focus on values of the stretch $\lambda \in[0.5,1]$. The restricted model considered in this section corresponds to the model developed in [11]. The results are strongly dependent on the choice of material parameters, as is shown in Fig. 11. Here the dependence $W(\lambda)$, according to (19), is plotted. With a decrease in $k_{i}=K_{i}^{c} / K_{i}^{\Delta}$, a new local minimum arises. By compressing the material from its reference state, $\lambda=1$, the strain energy increases until a certain point. After that, it decreases until it reaches a local minimum. In other words, the whole material softens and no force is needed for its compression. Still, the growth condition (42) is fullfilled and it is not in conflict with the behaviour observed.

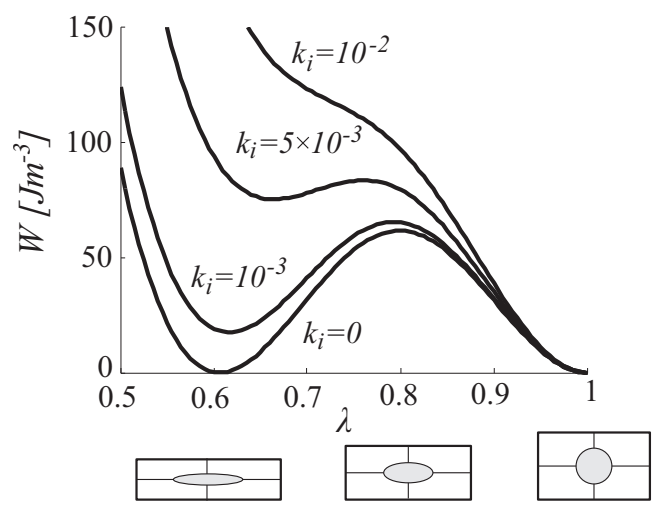

Fig. 11. Dependence of the strain energy function on compression of the macroscopic body for various values of $k_{i}$

Decreasing strain energy is here the result of non-affine deformations at the microscale, namely the deformation of inner volume. With $k_{i}$ decreasing, the inner volume is getting softer. In the limit case $k_{i} \rightarrow 0$ the inner volume has no elasticity and is in fact only a constraint (constant volume) for the springs of extracellular matrix. As the macroscopic body is deformed, considering macroscopic incompressibility, the shape and size of the RVE complies with the macroscopic deformation according to relations (33). The inner volume, on the other hand, takes the shape and size to minimize overall energy according to (38). In this particular case $\left(k_{i} \rightarrow 0\right)$ there exist a macroscopic deformation state $(\lambda=0.6)$ for which the inner volume takes such shape and size which vanishes the deformation energy. That is, it allows springs of extracellular matrix to reach their rest lengths. We consider that a non-affine deformation. We have no experimental evidence of such behaviour, it is rather a theoretical example to show the abilities of the model. This example illustrates non-linear mechanical response of the proposed model. The components of the model are linear, in principle. However, minimization of the overall energy at a given deformation state of a macroscopic body leads to the solution exhibiting high non-linearity. 


\subsection{Prestress}

There is experimental evidence that living cells may carry a pre-existing tension in the reference state, known as prestress [27]. This prestress may be included in this model by employing the idea of cell incompressibility. The springs within the embedded volume are assumed to be extended even in the reference state, as described in Section 3.2. The prestress parameters $P_{i}$ are introduced according to (25). Regarding a simple tension test, the Young modulus of the macroscopic body can be determined:

$$
Y \approx Y_{0}\left(1+f \cdot P_{1}\right)
$$

as is described in detail in $[32,33]$. Here, $Y_{0}$ is the Young modulus of the material with no prestress and $f$ is a function of the material parameters. The linear dependence obtained by the model is in agreement with experimental observations of the phenomenon known as prestress-induced stiffening [27].

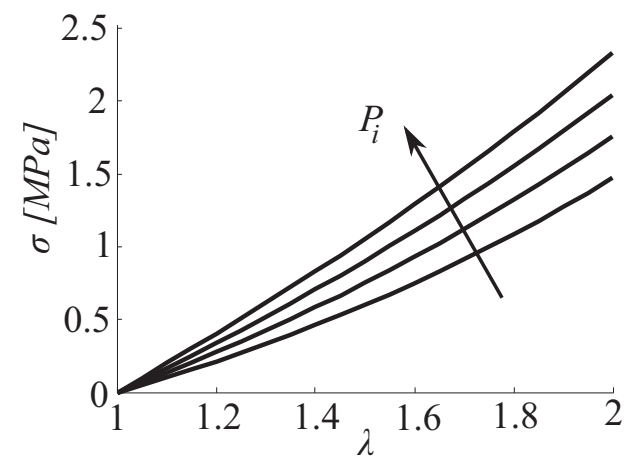

Fig. 12. Stress-stretch characteristics for various levels of prestress

The influence of the prestress on the macroscopic mechanical response is shown by plotting the stretch-stress characteristics of a simple tension test as in Fig. 12. Increasing the prestress, the material stiffens. Hence, this can be understood as a control mechanism of tissues to prevent extensive deformations. This model allows one to capture such an effect, i.e. to control the overall mechanical response by setting a certain parameter of the microstructure. For details, see [32].

\subsection{Modelling of real tissue}

An important advantage of the model is the transparent physical meaning of the material parameters. Some of them may be identified directly using microscopic measurements, as is shown in [35]. Here, a segment of a porcine carotid artery is loaded with an inner pressure $\Delta P$, causing its inflation. For this model, an arterial wall as a two-layer system composed of media and adventitia is used. Since the mechanical response of adventitia is driven by collagen fibrils oriented in two preferred directions, a structural model of [14] is used to define the strain energy function. The media, on the other hand, is mostly formed of smooth muscle tissue and hence our model is used to define its strain energy function. For details, see [35].

The microscopic measurements suggest a circumferential orientation of smooth muscle cells with the sizes

$$
c_{1}=c_{3}=6 \mu \mathrm{m}, \quad c_{2}=72 \mu \mathrm{m}, \quad \Delta_{i}=1.4 \mu \mathrm{m} .
$$

Using these values, the material parameters of the model related to the geometry are determined. Only the stiffness-like parameters are left to be identified using a least-squares fitting. The results 


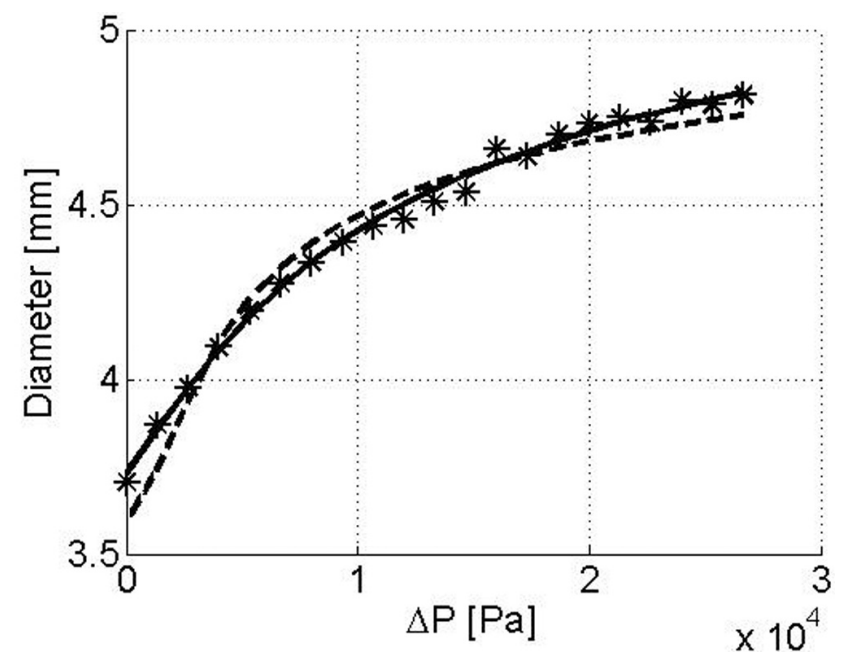

Fig. 13. Diameter $v s$ inner pressure in a porcine carotid artery. Experimental data (asterisks) and model predictions (solid line). A Holzapfel model (dashed line) is plotted for comparison

are depicted in Fig. 13. Here, the dependence of the diameter of the arterial ring on the inner pressure $\Delta P$ is plotted. The good agreement of the model with the experimental data may be due to the least-squares fitting. However, the identification of some of the material parameters directly from microscopic measurements, allowed by this model, certainly represents progress in the continuum description of soft tissues. An arterial ring treated using the Holzapfel model alone is plotted for comparison (dashed line).

\section{Conclusion}

This paper presents a two-scale hyperelastic model motivated by the arrangement of smooth muscle tissue. The notion of a representative volume element is employed to define a microstructure formed of linear elastic elements. The main focus of the paper is on anisotropy as an important feature governing the mechanical response of soft tissues, as well as many engineering materials. The anisotropy of the material is taken into account using the notion of structural tensors and pseudo-invariants. That is, the strain energy of the proposed material model is expressed in terms of pseudo-invariants, which is a convenient form for FE analyses $[14,20]$. First, an illustrative example is presented to demonstrate the effect of anisotropy on the mechanical response. On the basis of this example, a material model is proposed, the microstructure of which is formed of incompressible volumes and linear springs. The model has a cellular structure and includes prestress and possibly non-affine deformations. It can be appplied to focus on certain aspects of soft biological tissues or other anisotropic materials in the framework of a continuum description and FE analyses.

A key idea in the proposed model consists in considering the microstructure to occupy an energy-minimizing configuration represented here by the shape of an embedded volume. Hence, non-affine deformations at the microscale are included in the continuum description of the macroscopic body. This phenomenon is shown through a uniaxial compression. While the macroscopic body at a point $\boldsymbol{x}$ is deformed according to the deformation gradient $\mathbf{F}(\boldsymbol{x})$, the volume within an associated RVE occupies a shape which tends to allow the springs to gain their rest lengths. This leads to the softening of the material and the occurrence of a new natural state. A description of such behaviour is not possible using classical approaches that employ 
affine deformations $[9,10]$. Hence, this model is promising because it couples non-trivial effects at the microscale with the overall mechanical response of a macroscopic body.

Another advantage of the proposed model is the ease of implementing prestress in the reference state. The assumption is that a constant volume of living cells does not allow the reinforcing springs to relax. Constant volume balances the tension within the springs and hence represents an alternative idea to the struts in tensegrity models. The linear dependence of the stiffness on the prestress obtained in the model corresponds to experimental observations in living cells [27].

In the proposed model, a bottom-up approach is applied. This means starting at the microscale by defining a model of the microstructure, subsequently moving up to the macroscale, for which the strain energy function is derived. The anisotropy of the model results naturally from the definition of the microstructure. Also, material parameters which appear in the formula of the strain energy function have transparent physical meanings related to the microstructure. They express the geometry and the arrangement of the microstructure as well as the stiffnesses of the linear elastic elements representing fibres. Although there is a large number of material parameters in general, their number can be reduced by adopting additional assumptions, such as transverse isotropy, which is common in the case of soft tissues [20,22]. Due to their transparent meaning, some of the material parameters may be identified directly using microscopic measurements, as is shown in the example of a porcine carotid artery. This is an advantage compared to classical phenomenological models. For them, the identification of the parameters may sometimes be problematic and have to be done using the least squares method $[15,19,28]$.

The model presented is two-scale hyperelastic anisotropic. It is a generalization of the model proposed previously by this research group. In the present form, any deformation state, including shears, may be described, thanks to the additional diagonal springs within the representative volume element. However, there is no exact analytical solution of the minimization problem associated with the strain energy function. Hence, the description of the deformation state of a macroscopic body is accompanied by a numerical minimization at each integration point, which is time consuming. Finding an approximate analytical formula for the strain energy function is thus an object for further research.

\section{Acknowledgements}

The work has been supported by the project SGS-2016-059 and by the project NEXLIZ CZ.1.07/2.3.00/30.0038.

\section{References}

[1] Blemker, S.S., Pinsky, P.M., Delp, S.L., A 3D model of muscle reveals the causes of nonuniform strains in the biceps brachii, Journal of Biomechanics 38 (2005) 657-665. https://doi.org/10.1016/j.jbiomech.2004.04.009

[2] Bonet, J., Wood, R. D., Nonlinear continuum mechanics for finite element analysis, Cambridge University Press, Cambridge, 1997.

[3] Buehler, M.J., Atomistic and continuum modeling of mechanical properties of collagen: Elasticity, fracture and self-assembly, Journal of Materials Research 21 (2006) 1947-1961. https://doi.org/10.1557/jmr.2006.0236

[4] Chuong, C. J., Fung, Y. C., Three-dimensional stress distribution in arteries, Journal of Biomechanical Engineering 105 (1983) 268-274. https://doi.org/10.1115/1.3138417 
[5] Ciarlet, P. G., Mathematical elasticity. Volume I: Three-dimensional elasticity, North-Holland, Amsterdam, 1988.

[6] Deng, S. X., Tomioka, J., Debes, J. C., Fung, Y. C., New experiments on shear modulus of elasticity of arteries, American Journal of Physiology 266 (1994) H1-H10.

https://doi.org/10.1152/ajpheart.1994.266.1.H1

[7] Drago, A., Pindera, M. J., Micro-macromechanical analysis of heterogeneous materials: Macroscopically homogeneous vs periodic microstructures, Composites Science and Technology 67 (2007) 1243-1263.

https://doi.org/10.1016/j.compscitech.2006.02.031

[8] Fung, Y. C., Fronek, K., Patitucci, P., Pseudoelasticity of arteries and the choice of its mathematical expression, American Journal of Physiology 237 (1979) H620-H631. https://doi.org/10.1152/ajpheart.1979.237.5.H620

[9] Gates, T. S., Odegard, G. M., Frankland, S. J. V., Clancy, T. C., Computational materials: Multiscale modeling and simulation of nanostructured materials, Composites Science and Technology 65 (2005) 2416-2434. https://doi.org/10.1016/j.compscitech.2005.06.009

[10] Guo, X., Wang, J.B., Zhang, H.W., Mechanical properties of single-walled carbon nanotubes based on higher order Cauchy-Born rule, International Journal of Solids and Structures 43 (2006) 1276-1290. https://doi.org/10.1016/j.ijsolstr.2005.05.049

[11] Holeček, M., Moravec, F., Hyperelastic model of a material which microstructure is formed by "balls and springs", International Journal of Solids and Structures 43 (2006) 7393-7406. https://doi.org/10.1016/j.ijsolstr.2006.06.031

[12] Holzapfel, G. A., Nonlinear solid mechanics, Wiley, Chichester, England, 2000.

[13] Holzapfel, G. A., Gasser, T. C., A viscoelastic model for fiber-reinforced composites at finite strains: Continuum basis, computational aspects and applications, Computer Methods in Applied Mechanics and Engineering 190 (2001) 4379-4403. https://doi.org/10.1016/S0045-7825(00)00323-6

[14] Holzapfel, G. A., Gasser, T.C., Ogden, R. W., A new constitutive framework for arterial wall mechanics and a comparative study of material models, Journal of Elasticity 61 (2000) 1-48. https://doi.org/10.1023/A:1010835316564

[15] Horny, L., Zitny, R., Chlup, H., Strain energy function for arterial wall based on limiting fiber extensibility, ECIFMBE Proceedings 22, 2008, pp. 1910-1913.

[16] Ito, D., Tanaka, E., Yamamoto, S., A novel constitutive model of skeletal muscle taking into account anisotropic damage, Journal of Mechanical Behavior of Biomedical Materials 3 (2010) 85-93. https://doi.org/10.1016/j.jmbbm.2009.05.001

[17] Itskov, M., Aksel, N., A class of orthotropic and transversely isotropic hyperelastic constitutive models based on a polyconvex strain energy function, International Journal of Solids and Structures 41 (2004) 3833-3848. https://doi.org/10.1016/j.ijsolstr.2004.02.027

[18] Kanit, T., Forest, S., Galliet, I., Mounoury, V., Jeulin, D., Determination of the size of the representative volume element for random composites: Statistical and numerical approach, International Journal of Solids and Structures 40 (2003) 3647-3679. https://doi.org/10.1016/S0020-7683(03)00143-4

[19] Klitsch, S. M., Lotz, J. C., Application of a fiber-reinforced continuum theory to multiple deformations of the annulus fibrosus, Journal of Biomechanics 32 (1999) 1027-1036. https://doi.org/10.1016/S0021-9290(99)00108-6

[20] Kuhl, E., Garikipati, K., Arruda, E. M., Grosh, K., Remodeling of biological tissue: Mechanically induced reorientation of a transversely isotropic chain network, Journal of the Mechanics and Physics of Solids 53 (2005) 1552-1573. https://doi.org/10.1016/j.jmps.2005.03.002

[21] Limbert, G., A mesostructurally-based anisotropic continuum model for soft tissues — Decoupled invariant formulation, Journal of the Mechanical Behavior of Biomedical Materials 4 (2011) 1637-1657. https://doi.org/10.1016/j.jmbbm.2011.07.016 
[22] Limbert, G., Middleton, J., A transversely isotropic viscohyperelastic material: Application to the modeling of biological soft connective tissues, International Journal of Solids and Structures 41 (2004) 4237-4260. https://doi.org/10.1016/j.ijsolstr.2004.02.057

[23] Nierenberger, M., Remond, Y., Said, A., A new multiscale model for the mechanical behavior of vein walls, Journal of the Mechanical Behavior of Biomedical Materials 23 (2013) 32-43. https://doi.org/10.1016/j.jmbbm.2013.04.001

[24] Shearer, T., A new strain energy function for modelling ligaments and tendons whose fascicles have a helical arrangement of fibrils, Journal of Biomechanics 48 (2015) 3017-3025. https://doi.org/10.1016/j.jbiomech.2015.07.032

[25] Spencer, A. J. M., Theory of invariants, in: Continuum Physics, ed. A.C. Eringen, Volume I, Academic Press, New York, 1971. https://doi.org/10.1016/B978-0-12-240801-4.50008-X

[26] Spencer, A.J.M., Soldatos, K.P., Finite deformations of fibre-reinforced elastic solids with fibre bending stiffness, International Journal of Non-Linear Mechanics 42 (2007) 355-368. https://doi.org/10.1016/j.ijnonlinmec.2007.02.015

[27] Stamenović, D., Wang, N., Ingber, D. E., Tensegrity architecture and the mammalian cell cytoskeleton, In: Multiscaling in molecular and continuum mechanics: Interaction of time and size from macro to nano, ed. G. C. Sih, Springer, Dordrecht, 2007, p. 321-338. https://doi.org/10.1007/978-1-4020-5008-4_14

[28] Takamizawa, K., Hayashi, K., Strain energy density function and uniform strain hypothesis for arterial mechanics, Journal of Biomechanics 20 (1987) 7-17. https://doi.org/10.1016/0021-9290(87)90262-4

[29] Tang, H., Buehler, M.J., Moran, B., A constitutive model of soft tissue: From nanoscale collagen to tissue continuum, Annals of Biomedical Engineering 37 (6) (2009) 1117-1130. https://doi.org/10.1007/s10439-009-9679-0

[30] Tonar, Z., Markoš, Z., Microscopy and morphometry of integument of the foot of pulmonate gastropods Arion rufus and Helix pomatia, Acta Veterinaria Brno 73 (2004) 3-8.

[31] Vaishnav, R. N., Young, J. T., Patel, D. J., Distribution of stresses and of strain-energy density through the wall thickness in a canine aortic segment, Circulation Research 32 (1973) 577-583. https://doi.org/10.1161/01.RES.32.5.577

[32] Vychytil, J., Holeček, M., Grid continuum description: A novel approach in two-scale hyperelasticity, Journal of Multiscale Modelling 4 (2012) 1250004. https://doi.org/10.1142/S1756973712500047

[33] Vychytil, J., Holeček, M., The simple model of cell prestress maintained by cell incompressibility, Mathematics and Computers in Simulation 80 (2010) 1337-1344.

[34] Vychytil, J., Holeček, M., Moravec, F., Prestress in "balls and springs" model, Applied and Computational Mechanics 1 (1) (2007) 363-370.

[35] Vychytil, J., Kochová, P., Tonar, Z., Kuncová, J., Švíglerová, J., Constitutive modelling of an arterial wall supported by microscopic measurements, Applied and Computational Mechanics 6 (1) (2012) 107-118.

[36] Wu, P.D., van der Giessen, E., On improved 3-D non-Gaussian network models for rubber elasticity, Mechanics Research Communications 19 (5) (1992) 427-433.

https://doi.org/10.1016/0093-6413(92)90021-2 